\title{
Perception of two crossing frequency glides
}

\author{
Michinao Matsui, ${ }^{*}+K_{\text {Kenji Kurakata, }}{ }^{* *}$ and Atsushi Nishimura*** \\ *Faculty of Language and Culture, Osaka, University, \\ 1-8, Machikaneyama, Toyonaka, 560-0043 Japan \\ ** National Institute of Bioscience and Human-Technology, MITI, \\ 1-1, Higashi, Tsukuba, 305-8566 Japan \\ *** Faculty of Home Economics, Mukogawa Women's University, \\ 6-46, Ikebiraki, Nishinomiya, 663-8558 Japan \\ (Received 12 December 1997)
}

Keywords : Frequency glide, Perception of frequency change, Gestalt principles, Good continuity, Continuity illusion

PACS number: $43.66 . \mathrm{Hg}, 43.66 . \mathrm{Lj}, 43.66 . \mathrm{Mk}$

\section{Introduction}

When we look at a drawing such as the one shown in Fig. 1(a), we tend to interpret it as two straight lines crossing [Fig. 1(b)], not as, for example, a wide V-shape and a second similar V-shape upside-down in contact with each other at the midpoints [Fig. 1(c)]. This is an example of the principle of good continuity in the Gestalt psychology of perception; the visual system prefers "good," "continuous" forms.

If we think of Fig. 1(a) as a pattern on a spectrogram, it could be heard as a sound consisting of sinusoidal glides. Several researchers have used this pattern of sound as stimulus (e.g., Halpern, 1977; Tougas and Bregman, 1990 ; McPherson et al., 1994) and investigated which percept was preferred, crossing [Fig. 1(b)] or bouncing [Fig. 1(c)]. They showed that in contrast to vision, the subjects favored the bouncing percept in audition. Bregman suggested that this is "because the principle of frequency proximity favors the grouping of glides that stay in the same frequency region" (Bregman, 1990, p.364). Deutsch's scale illusion (Deutsch, 1975) is another example regarded as being affected by the same principle, although the sounds used are discrete and dichotically presented.

In a series of experiments concerning continuity illusion where gliding tones were interrupted by a noise, Kurakata et al. $(1994,1996)$ showed evidence that the auditory system traced the frequency change of the pre-noise glide and extrapolated the glide into the following noise. Trajectory continued to be extrapolated during the noise up to a maximum of about $120 \mathrm{~ms}$. This frequency-extrapolation mechanism seems to underlie the continuity illusion with gliding tones.

As an explanation for this phenomenon, Matsui et al. (1995; see also Kurakata et al., 1997) proposed a two-stage model of auditory processing. In the model they assumed that the auditory system first seeks pos-

\footnotetext{
${ }^{\dagger}$ Now at Institute for Linguistic Sciences, Kobe Shoin Women's University
}

sible trajectories in a time window of about $100 \mathrm{~ms}$, then the candidates are successively connected over time according to the principle of frequency proximity.

If the extrapolation mechanism works also for sounds like that represented in Fig. 1(a), and the effect is stronger than that of frequency proximity, then it would elicit a crossing percept of two glides, not a bouncing percept as reported in the previous studies. This phenomenon would be typically observed when the duration is equal to or shorter than the time window of about $100 \mathrm{~ms}$. To examine this hypothesis and an interaction of the extrapolation mechanism with the effect of frequency proximity the following experiment was conducted.

\section{Experiment}

\subsection{Method}

The stimuli consisted of two sinusoidal glides with logarithmically changing frequency. The frequency change was either in opposite directions [the oppositedirection condition; Fig. 2(a)] or in the same direction [the same-direction condition; Fig. 2(b)].

In the opposite-direction condition, a descending glide changed from 1,414 to $707 \mathrm{~Hz}$ and an ascending glide changed from 707 to $1,414 \mathrm{~Hz}$. The duration of the glides was either 100,500 or $1,000 \mathrm{~ms}$ with rise and fall times of $20-\mathrm{ms}$ cosine ramps. The two glides crossed at $1,000 \mathrm{~Hz}$ at the midpoints of the various durations.

In the same-direction condition, one glide (the steeper glide) changed from 2,000 to $500 \mathrm{~Hz}$ and the other glide (the shallower glide) changed from 1,500 to 750 $\mathrm{Hz}$. The duration of the glides in this condition was fixed at 1,000 ms with rise and fall times of 20-ms cosine ramps. The two glides crossed near the midpoints of their durations.

The sound pressure level of the glides was $58 \mathrm{~dB}$. The presented level was adjusted using a measuring amplifier (B\&K, type 2610) and an artificial ear (B\&K, type 4153 ) with an adapter (B\&K, DB 0843).

These stimuli were presented to subjects in an an- 
(a)

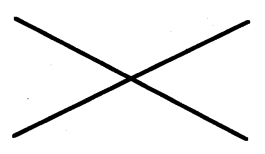

(b)

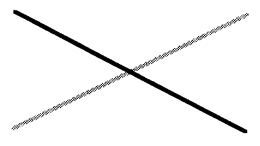

(c)

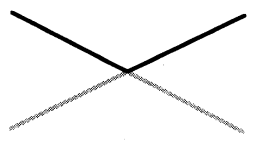

Fig. 1 An example of the principle of good continuity in the Gestalt psychology of visual perception. Panels (b) and (c) are two possible percepts for the figure in panel (a).

(a)

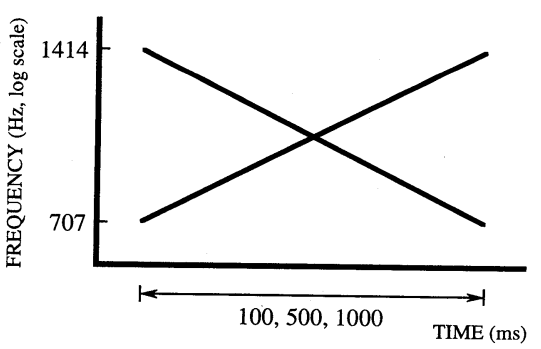

(b)

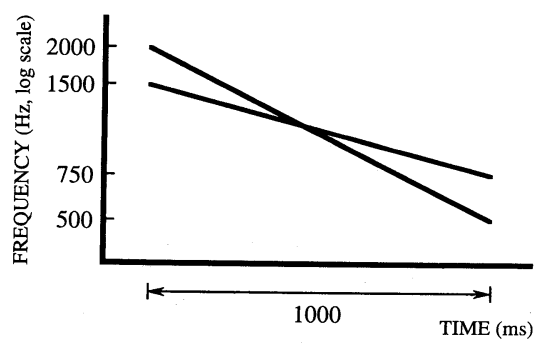

Fig. 2 Schematic illustrations of the experimental stimuli. (a) Opposite-direction condition,

(b) same-direction condition.

echoic room via headphones (STAX, SR- $\Lambda$ pro). The stimuli of both direction conditions were randomly presented 10 times for each stimulus. Subjects were instructed to pay attention only to one of the two glides. Before each trial, a steady-state tone with the same frequency as the initial of one of the two glides was presented to indicate which glide the subjects should pay attention to. The duration of the indicating tone was $1 \mathrm{~s}$. After listening to each stimulus, they were required to answer whether the glide was "crossing" with the other glide or "bouncing" at the midpoint by choosing a picture. The pictures used are shown in Fig. 3. Panels (a) and (b) were used for judging a (a)

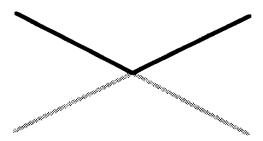

(b)

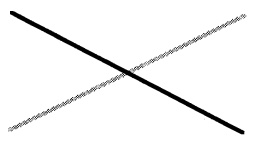

(c)

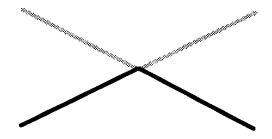

(d)

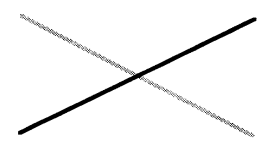

Fig. 3 Possible alternative perceptions for the stimuli used in the experiment. (a) Descending-bouncing, (b) descendingcrossing, (c) ascending-bouncing, (d) ascending-crossing.

descending glide and panels (c) and (d) for an ascending glide (the same pictures were also used for the same-direction condition, to avoid the subjects being confused by the two conditions). They could listen to the stimuli as many times as they liked.

Eight subjects in their twenties participated in the experiment. They all had normal hearing ability.

2.2 Results and discussion

2.2.1 Opposite-direction condition

Table 1 shows the selection percentages for crossing and bouncing perceptions in both direction conditions. In the opposite-direction condition, every subject perceived the 500- and 1,000-ms glides in all trials as bouncing. This result is consistent with those reported in the literature cited above.

On the other hand, for the 100-ms glides some perception of crossing was obtained, as Matsui's model predicts. When the subjects traced the descending glide, the perception of bouncing was still dominant (binomial test ; $z=3.02, p<.01$ ), although there was no significant dominance for the ascending glide $(z=.34, p>.05)$. Further informal experiments where the initial and final frequencies of the glides were not in simple ratios showed the same tendency. This indicates that the simple frequency ratio of both ends between two glides is not essential for the crossing percept.

The perception of bouncing has been explained in terms of the principle of frequency proximity. However, when the duration of glides is short enough, the effect of the frequency-extrapolation mechanism seems to overcome that of frequency proximity and elicits the perception of crossing. 


\section{MATSUI et al. : PERCEPTION OF TWO FREQUENCY GLIDES}

Table 1 Selection percentages for crossing and bouncing perceptions in the oppositedirection condition and the same-direction condition.

\begin{tabular}{|c|c|c|c|c|c|}
\hline \multirow{2}{*}{ Direction of glides } & \multirow{2}{*}{ Duration of glides (ms) } & \multicolumn{2}{|c|}{ Descending } & \multicolumn{2}{|c|}{ Ascending } \\
\hline & & Bouncing & Crossing & Bouncing & Crossing \\
\hline \multirow{3}{*}{ Opposite } & 1,000 & 100.0 & 0.0 & 100.0 & 0.0 \\
\hline & 500 & 100.0 & 0.0 & 100.0 & 0.0 \\
\hline & 100 & 67.5 & 32.5 & 52.5 & 47.5 \\
\hline \multirow{2}{*}{ Direction of glides } & \multirow{2}{*}{ Duration of glides (ms) } & \multicolumn{2}{|c|}{ Steeper } & \multicolumn{2}{|c|}{ Shallower } \\
\hline & & Bouncing & Crossing & Bouncing & Crossing \\
\hline Same & 1,000 & 35.0 & 65.0 & 20.0 & 80.0 \\
\hline
\end{tabular}

Furthermore, McPherson et al. (1994) have pointed out that two frequency glides that swept through the same frequency range in opposite directions could cross perceptually if their rates of change differed, as suggested by analogous visual perception. The results of the present experiment show, however, that a crossing perception can be elicited even if the two glides change frequency at the same rate.

The duration of $100 \mathrm{~ms}$ may seem too short for the human auditory system to track in rapid frequency change. Steiger (1980) has suggested, however, that glides could be distinguished from steady-state tones even when their durations were as short as $50 \mathrm{~ms}$. "This proves that the measurements made by the auditory system on the slope of a glide can be completed in 50 ms" (Bregman, 1990, p.113). Although the slope of the glides in the present experiment was steeper than in Steiger's experiment, it might be possible for the subjects to judge the property of the glides.

There is one point which is still unclear from the present experiment. That is the effect of the slope of the glides, which was not controlled. in this experiment. Since the range of frequency change was always kept at one octave, the slope of the glide became steeper as the duration was made shorter. Thus, the steepness might have favored the perception of crossing. This manipulation of the stimuli was inevitable to keep the range of frequency change constant and to avoid unnecessary effects of "energy splatter" at the onset and offset of the glides which might have smeared the frequency components around the intersection. Further experiments will be required to evaluate the effect of the slope using another set of stimuli.

\subsubsection{Same-direction condition}

In the same-direction condition, the perception of crossing was more dominant than that of bouncing (binomial test ; $z=2.57, p<.01$, for steeper glide; $z=$ $5.25, p<.01$, for shallower glide). Similar results were obtained when one of the glides was flat (i.e., it did not change in frequency). Therefore, when changes in frequency for the two glides proceed in the same direction, the effect of frequency proximity becomes weaker than that of tracing the frequency changes and a perception of crossing is obtained.

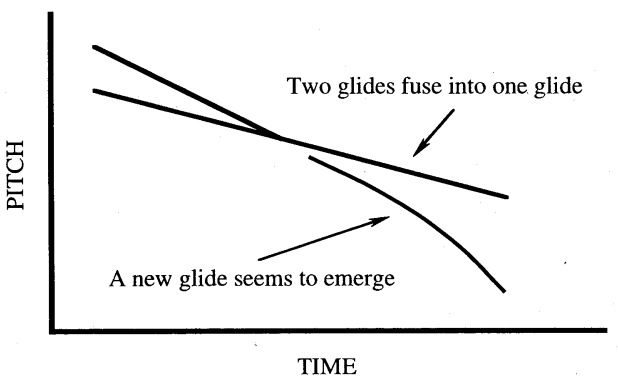

Fig. 4 Another possible percept for the stimulus in Fig. 2(b).

Some subjects reported, based on their retrospection, that they perceived the two glides as fusing into one glide at the intersection and a new lower-pitched glide appearing, as shown in Fig. 4. When two tones with similar frequencies are presented simultaneously, beat or relatively long temporal gaps can generally be produced by their interaction. They were also observed in the waveform of the present stimulus. These beat or gaps might have some effect on this percept, although they are not clearly audible and cannot explain completely why the two glides fuse into a higher glide. There may be another unknown factor which determines the perception of two coexistent glides changing in the same direction.

\section{Conclusions}

These experimental results show that when two gliding tones cross each other, they can create a crossing percept, as well as the bouncing percept suggested in the previous literature. This effect could be observed when the glide durations were as short as $100 \mathrm{~ms}$. It seems the effect of the frequency-extrapolation mechanism is more dominant than that of frequency proximity within this short time span. Furthermore, the frequency changes of the glides were easily traced when they did not change in opposite directions, and a crossing perception could be elicited. 
Acknowledgment

One of the authors, Kurakata, was supported by a Grant-in-Aid for JSPS Fellows to conduct this study. The authors are grateful to an anonymous reviewer for useful comments on an earlier version of the paper.

\section{References}

Bregman, A. S. (1990). Auditory Scene Analysis: The Perceptual Organization of Sound (MIT Press, Cambridge, MA).

Deutsch, D. (1975). "Two-channel listening to musical scales," J. Acoust. Soc. Am. 57, 1156-1160.

Halpern, L. (1977). "The effect of harmonic ratio relationships on auditory stream segregation," Unpublished research report, Psychology Department, McGill University.

Kurakata, K., Matsui, M., and Nishimura, A. (1994). "Perceptual trajectory of the continuity effect of frequency glide," Trans. Tech. Comm. Psychol. Physiol. Acoust. H-94-71 (in Japanese with English abstract).

Kurakata, K., Matsui, M., and Nishimura, A. (1996). "Perceptual trajectory of gliding tones through interrupting noise and the underlying frequency-extrapolation mechanism," Proc. 3rd Jt. Meet. ASA/ASJ, 695-700.

Kurakata, K., Matsui, M., and Nishimura, A. (1997). "A cognitive model of auditory scene analysis," Proc. 1st Int. Conf. on Cognitive Science, 183-187.

McPherson, L., Ciocca, V., and Bregman, A. (1994). "Organization in audition by similarity in rate of change: Evidence from tracking individual frequency glides in mixtures," Percept. Psychophys. 55, 269-278.

Matsui, M., Kurakata, K., and Nishimura, A. (1995). "Psychological processes of prediction and constraints in auditory scene analysis," Tech. Rep. IEICE, SP95-44, 17-24 (in Japanese with English abstract).

Steiger, H. (1980). "Some informal observations concerning the perceptual organization of patterns containing frequency glides," Unpublished report, McGill University.

Tougas, Y. and Bregman, A. S. (1990). "Auditory streaming and the continuity illusion," Percept. Psychophys. 47, $121-126$

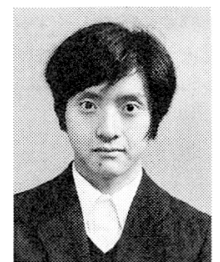

Michinao Matsui graduated from Department of Human Sciences, Osaka University. He received his master's degree (Language and Culture) from Osaka University in 1992, and his doctor's degree (Language and Culture) from Osaka University in 1998. He is now a lecturer at the Institute for Linguistic Sciences, Kobe Shoin Women's University. His research areas have been in psychoacoustics, speech perception, language processing, generative phonology and generative grammar.

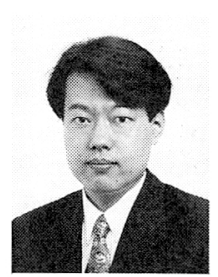

Kenji Kurakata was born in Tokorozawa, Japan in $1967 . \mathrm{He}$ received his B. A. degree from Waseda University in 1989 and his M. A. and $\mathrm{Ph} . \mathrm{D}$. degrees from Osaka University in 1991 and 1994, respectively, in psychoacoustics. He was a Research Fellow of the Japan Society for the Promotion of Science at Osaka University and the Nara Institute of Science and Technology from 1991 to 1996. $\mathrm{He}$ is currently a researcher at the National Institute of Bioscience and Human-Technology, MITI, Japan. His research interests are the cognitive model of auditory processing, human-computer interface with auditory signals, and auditory perception of the elderly. He received the Awaya Prize from the Acoustical Society of Japan in 1996.

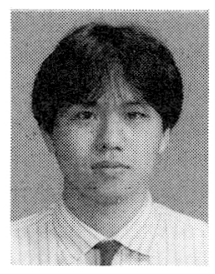

Atsushi Nishimura received his bachelor's degree (Science of Art) from Osaka University of Arts in 1991 and his master's degree (Home Economics) from Mukogawa Women's University in 1996. He is now involved in research and design of sound archives for soundscape studies. 